\title{
Multiplet Splitting for the XPS of Heavy Elements: Dependence on Oxidation State
}

Paul S. Bagus, ${ }^{a}$ Connie J. Nelin, ${ }^{b}$ Yahya M. Al-salik, ${ }^{c}$ Eugene S. Ilton ${ }^{d}$ and Hicham Idriss. ${ }^{c}$

${ }^{a}$ Department of Chemistry, University of North Texas, Denton, TX 76203-5017, USA

${ }^{\mathrm{b}}$ Consultant, Austin, TX 78730, USA

${ }^{\mathrm{C}} \mathrm{SABIC}$, Corporate Research Institute (KAUST), Saudi Arabia

${ }^{\mathrm{d}}$ Pacific Northwest National Laboratory, Richland, WA 99352, USA

\begin{abstract}
Multiplet splittings in X-Ray Photo-electron Spectroscopy, XPS, are a means of distinguishing different open shell occupations, or different oxidation states, in a material being studied. Indeed, especially for $3 \mathrm{~d}$ transition metal complexes, they have provided fingerprints of the metal oxidation state. The present work provides theoretical and experimental evidence that it may also be possible to use multiplets to characterize the oxidation state of heavy metal, lanthanide and actinide, cations in complexes. However, it is important to make a proper choice of the XPS region to study in order to obtain large multiplet splittings. We identify a low binding energy, BE, peak that had been observed for $\mathrm{Ce}(\mathrm{III})$ in $\mathrm{CeO}_{\mathrm{x}}$ as a high spin coupled multiplet. Furthermore, we show that a low $\mathrm{BE}$ feature with reasonable intensity is characteristic of other XPS regions and of other metals. This feature arises from a high spin multiplet and serves as a fingerprint to distinguish closed shell from open shell cations. Evidence is presented that it may also be possible to distinguish different open shell occupations.
\end{abstract}

Keywords: XPS, Heavy metal atoms, Multiplets, Intermediate Angular Momentum Coupling

\section{Introduction}

The X-Ray photoelectron spectroscopy, XPS, of 3d transition metal complexes often contain several features that arise from the presence of a localized open shell $3 \mathrm{~d}$ electrons. The existence of these features, their origin in the electronic structure of the material, and their potential to be used to distinguish different oxidation states has been known for some time; see, for example, Refs. [1-11]. In part, these features arise from angular momentum coupling and recoupling within the $3 \mathrm{~d}$ shell and between the $3 \mathrm{~d}$ shell and the open core shell in the final corehole configuration. These couplings give rise to different degenerate sets of states, or multiplets, in the core-hole systems and the XPS structure is described as a multiplet structure. Since a 
driving force for the energy separation of the multiplets is the exchange interaction between the core-hole with the open nd shell, in the case of transition metals, or nf shell, in the case of lanthanides and actinides, [3, 12-13] these splittings have also been called exchange splittings. [14] In addition to the angular momentum coupling, there are other atomic many-body effects that involve moving electrons between shells with the same principle quantum number, which are especially important for the $3 \mathrm{~s}$ holes of $3 \mathrm{~d}$ transition metal cations. [5, 15] The term multiplets is also used to include these atomic effects since they allow new, normally forbidden, multiplets to get XPS intensity.

While multiplets are regularly used to describe the XPS of $3 \mathrm{~d}$ ionic compounds and even of $3 \mathrm{~d}$ metals, [16] they are generally not used to describe the XPS of heavy metals, such as lanthanides and actinides. Instead, the focus for these systems is on satellite structure [17-18] that arise from many-body effects involving the interaction between the cation and the ligands. [1820] In part, this is because the most intense XPS is for the $3 \mathrm{~d}$ levels of lanthanides and the $4 \mathrm{f}$ levels of actinides, which have small multiplet splittings; for the multiplet splittings in U cations, see, for example, Ref. [21]. However, large multiplet splittings are very likely to be associated with ionization of a subshell with the same principle quantum number as the open outer shell of the metal cation. Thus for lanthanides, the $4 p$ and $4 d$ may have large multiplet splittings from coupling with the open $4 \mathrm{f}$ shell and, for actinides, the $5 \mathrm{p}$ and $5 \mathrm{~d}$ may have large multiplet splittings from coupling with the open $5 \mathrm{f}$ shell. The $4 \mathrm{~s}$ and $5 \mathrm{~s}$ shells are neglected because their XPS intensity will be considerably smaller than for the np and nd shells. The reason for the larger multiplet splittings between shells that have the same principle quantum number comes from the magnitude of the exchange interaction between the core and open valence shells. In general, the exchange integrals, which are a measure of the exchange interaction, are larger between shells 
that have similar spatial extents. [22]. As a result, it may be possible to distinguish oxidation states of heavy metals as previously demonstrated for the $2 \mathrm{p}$ XPS of the $3 \mathrm{~d}$ transition metals. [12] For example, Mullins et al. [18] identified a low binding energy, BE, peak in the Ce $4 \mathrm{~d}$ spectra as an indicator, or fingerprint, of the presence of $\mathrm{Ce}^{3+}$. In the present work, this feature is shown to be the lowest energy multiplet from the angular momentum coupling of the $4 \mathrm{~d}$ hole with the $4 \mathrm{f}^{1}$ occupation in $\mathrm{Ce}^{3+}$. We also show that similar fingerprints of the oxidation state exist for the other spectral regions considered. In this paper, we shall study multiplet splitting for the $4 \mathrm{p}$ and 4d XPS of Ce and the 5p and 5d XPS of U. For each of the metals, we will show the potential for the multiplet splittings to distinguish among oxidation states.

In the following section, Sec. II, we discuss the materials models ( free ions or embedded small clusters), the wavefunctions and the methods for modeling the XPS spectra from the calculated wavefunctions. We also describe how the experimental XPS spectra for $\mathrm{U}$ doped $\mathrm{CeO}_{2}$ and $\mathrm{UO}_{2}$ were obtained. In Sec. III, we examine the predicted XPS for different charge states of $\mathrm{Ce}$ and $\mathrm{U}$ cations and show that the spectra do, indeed, clearly reflect the charge state of the ion. Moreover, we examine the composition of some of the features of the spectra in term of RussellSaunders, RS, multiplets and assess the importance of ligand field splittings by comparing the XPS spectra predicted for atomic and for embedded cluster models. In Sec. IV, we compare our results with experiment and show that the predicted multiplet splittings are, indeed, observed in Ce and U XPS spectra. Finally in Sec. V, we summarize the evidence that supports our principle conclusion that multiplet splittings in the XPS of outer core shells provide an alternative approach to determine the oxidation states of heavy metal ions. 


\section{Theoretical and Experimental Methods}

\section{A. Materials Models, Theoretical Concepts and Theoretical Methods}

We have used two materials models. The first model is the isolated cation, which allows us to study the multiplet splittings independent of ligand field or other condensed phase effects. The second is an embedded cluster model of the fluorite structures of $\mathrm{CeO}_{2}$ and $\mathrm{UO}_{2}$, which tests potential ligand field effects on the calculated XPS spectra. The initial and final, core ionized states are described by wavefunctions, WF's, which provide a natural framework for treating the angular momentum coupling of the open shells. In particular, the coupling will be intermediate between the limits of Russell-Saunders, RS, coupling where the total orbital, L, and spin, S, are each good quantum numbers and couple to each other to give a total $\mathrm{J}$ and $\mathrm{j}-\mathrm{j}$ coupling where the spin-orbit coupling is at the orbital level. The WF's are combinations of determinants formed from four component spinors, which are variational Dirac Hartree-Fock solutions for the average of configurations [23] for the initial state and the core-hole state configurations. It is important to stress that the individual many-electron determinants formed from these spinors are not normally, by themselves, eigenfunctions of the angular momentum operators and it is necessary to use combinations of determinants to obtain these eigenfunctions. For isolated atoms these eigenfunctions, which are linear combinations of determinants, are denoted $\mathbf{J}$ and $\mathrm{J}_{\mathrm{z}}$, but for the clusters, the irreducible representations of the appropriate point groups are used. The method that we use to determine these eigenfunctions is to take linear combinations of determinants where the open shell electrons are distributed in all possible ways over the spinors within that shell with the constraint that the occupied spinors belong to the initial or final state configuration. The mixing of determinants to obtain the total N-electron WF's are described as restricted complete open shell configuration interaction, COSCI, WF's or, for short, simply CI WF's. These CI 
wavefunctions are determined as solutions of a Hamiltonian matrix over the individual determinants where there is only one matrix for all the different angular momentum coupled states that may arise for the particular configuration. [24] With this approach, we naturally and rigorously take account of the intermediate coupling of the electrons between $\mathrm{j}-\mathrm{j}$ and $\mathrm{RS}$ coupling. This coupling will be examined in detail for the atomic models.

It is natural to think of angular momentum coupling in terms of $\mathrm{RS}$ coupled ${ }^{2 \mathrm{~S}+1} \mathrm{~L}_{\mathrm{J}}$ multiplets [22] where this coupling may give insights into the nature and origin of satellites. In particular, the decomposition of the spin-orbit coupled levels where only $\mathbf{J}$ is a good quantum number into contributions from different RS multiplets [25] is considered in detail for the atomic models discussed in the following section. We obtain wavefunctions for these RS multiplets by raising the speed of light for the calculation of the spinors and COSCI WF's. In the limit of $c \rightarrow \infty$, the solutions of the relativistic equations reduce to the non-relativistic case for spinors and COSCI WF's. We have chosen a value of $c=7500$ atomic units, or $>50$ times the correct value of $\mathrm{c}=137$ a.u., where the spin-orbit splittings for spinors and total WF's are reduced to $\sim 0.001 \mathrm{eV}$. These RS multiplet WF's are projected on to the fully relativistic intermediate coupled WF's to determine the population of the relativistic levels in terms of RS multiplets.

The XPS intensities are determined using the sudden approximation, SA, $[11,26]$ where the relative XPS intensity, $\mathrm{I}_{\text {rel }}$, to a given final state WF is taken as the overlap between the initial state WF where a core-electron has been annihilated and the final, ionized WF. A difficulty for the calculation of these matrix elements arises because there are different sets of orbitals, one optimized for the initial state configuration and the other for the final, core-hole configuration. As a consequence, we had to evaluate determinants of suitable overlap integrals between the initial 
and final state spinors. [11, 27] In order to be able to compare our theoretical XPS intensities with experimental spectra, we broaden our results to take account of experimental resolution and of lifetime broadening. Although a Voigt convolution of a Lorentzian, for lifetime broadening, and a Gaussian, for experimental resolution, is most accurate, [28] we generally approximate the broadenings with only a Gaussian.

For the isolated $\mathrm{Ce}$ species, we obtained wavefunctions for closed shell $\mathrm{Ce}^{4+}$, $\ldots . .4 \mathrm{p}^{6} 4 \mathrm{~d}^{10} 4 \mathrm{f}^{0} 5 \mathrm{~s}^{2} 5 \mathrm{p}^{6}$ and for open shell $\mathrm{Ce}^{3+}$ with occupation $4 \mathrm{f}^{1}$. The XPS regions considered were for $4 p$, and $4 d$ core-holes. For the isolated $U$ species, we considered the open shell species $\mathrm{U}^{+4}\left(5 \mathrm{f}^{2}\right)$ and $\mathrm{U}^{+5}\left(5 \mathrm{f}^{1}\right)$ with configurations .....5 $\mathrm{p}^{6} 5 \mathrm{~d}^{10} 5 \mathrm{f}^{\mathrm{n}} 6 \mathrm{~s}^{2} 6 \mathrm{p}^{6}$. For the XPS, $5 \mathrm{p}$ and $5 \mathrm{~d}$ hole configurations were considered. As models for $\mathrm{CeO}_{2}$ and $\mathrm{UO}_{2}$, we used embedded $\mathrm{XO}_{8}$ clusters shown schematically in Fig. 1. The clusters contain a central metal cation and it eight nearest neighbor oxygen anions. These 9 atoms are embedded in point charges of +4 , representing cations and -2 , representing anions, placed at lattice sites. Three shells of point charges were used for the embedding. Lattice constants appropriate for $\mathrm{UO}_{2}$ and $\mathrm{CeO}_{2}$ were used. [29] The oxidation state of $\mathrm{U}$ in $\mathrm{UO}_{8}$ is $\mathrm{U}(\mathrm{IV})$ with 2 electrons in an open shell. The oxidation state of Ce in $\mathrm{CeO}_{8}$ was taken as $\mathrm{Ce}(\mathrm{III})$ with 1 electron in an open shell even though the geometry and coordination of the cluster is for Ce(IV). This choice is reasonable since it has been shown that reduced Ceria in $\mathrm{CeO}_{2}$ has a local geometric environment essentially the same as that of pure $\mathrm{CeO}_{2}$ and that the defects that lead to the reduction to $\mathrm{Ce}(\mathrm{III})$ are distant from the reduced $\mathrm{Ce}$. [30] More details of these cluster models are given elsewhere. [19, 31]

The basis sets used to describe the isolated cations and the clusters are as used in previous studies. [19, 31] The orbitals, spinors, and WF's were computed with the Dirac program system 
[32] modified to interface with programs for the calculation of occupation numbers as well as of many-electron overlap and dipole transition matrix elements. These matrix elements were computed using a formalism based on cofactors [33-34] as implemented in the CLIPS program [35] interfaced to Dirac.

\section{B. XPS Measurements and materials synthesis}

$\mathrm{CeO}_{2}, \mathrm{UO}_{2}$ and $\mathrm{Ce}_{\mathrm{x}} \mathrm{U}_{1-\mathrm{x}} \mathrm{O}_{2}$ were synthesized by the precipitation method from their precursors following the procedure explained in Ref. [36]. The XPS spectra were taken using a Thermoscientific ESCALAB $250 \mathrm{Xi}$, equipped with a mono-chromated AlK $\square$ X-ray source, Ultra Violet He lamp for UPS, ion scattering spectroscopy (ISS), and reflected electron energy loss spectroscopy (REELS) was used. The base pressure of the chamber was typically in the low $10^{-10}$ mbar range. Charge neutralization was used for all samples (compensating shifts of $\sim 1 \mathrm{eV}$ ). Spectra were calibrated with respect to $\mathrm{C} 1 \mathrm{~s}$ at $284.7 \mathrm{eV}$. The U4f, U4d, U5p, Ce3d, Ce4d, Ce 4p, $\mathrm{O} 1 \mathrm{~s}, \mathrm{C} 1 \mathrm{~s}$ and valence band binding energy regions were scanned for all $\mathrm{Ce}_{\mathrm{x}} \mathrm{U}_{1-\mathrm{x}} \mathrm{O}_{2}$ materials. Typical acquisition conditions were as follows: pass energy $=20 \mathrm{eV}$ and scan rate $=0.1 \mathrm{eV}$ per 200ms. Ar ion bombardment was performed with an EX06 ion gun at $1 \mathrm{kV}$ beam energy and 10 mA emission current; sample current was typically 0.9-1.0 $\mu$ A. The sputtered area of $900 \times 900$ $\mu \mathrm{m}^{2}$ was larger than the analyzed area: $600 \mathrm{x} 600 \mu \mathrm{m}^{2}$. Self-supported oxide disks of approximately $0.5 \mathrm{~cm}$ diameter were loaded into the chamber for analysis. Data acquisition and treatment was done using the Avantage software. 


\section{XPS for Atomic Models}

The $4 \mathrm{~d}$ and $3 \mathrm{~d}$ XPS of $\mathrm{Ce}^{+4}$ and $\mathrm{Ce}^{+3}$ are shown in Fig. 2. The calculated energies are broadened with a Gaussian of $1.5 \mathrm{eV} \mathrm{FWHM}$ and the energy scale, $\mathrm{E}_{\text {rel }}$, is set to 0 for the lowest energy of the states for the core-hole configurations. The same choice of origin is made for all of the other plots in this paper. Fig. 2 illustrates the importance of the open shell occupation, or charge state, and of the ionized shell on the XPS. Of course, other many-body effects besides angular momentum coupling may be important, especially for the XPS of Cerium oxides, [31, 37] and these will be considered later. The $4 \mathrm{~d}$ XPS of closed shell $\mathrm{Ce}^{+4}$, Fig. 2(a), is a doublet with the higher $\mathrm{BE} 4 \mathrm{~d}_{3 / 2}$ peak spin-orbit split from the $3 \mathrm{~d}_{5 / 2}$ peak by $3.5 \mathrm{eV}$. The intensity ratio $I_{\text {rel }}\left(3 d_{5 / 2}\right) / I_{\text {rel }}\left(3 d_{5 / 2}\right)=1.67$ reflects the occupations of the $3 d_{5 / 2}$ and $3 d_{3 / 2}$ shells. The $3 d$ XPS, shown in Fig. 2(b), is similar to the 4d XPS with the exception that the spin-orbit splitting of $3 d_{5 / 2}$ and $3 \mathrm{~d}_{3 / 2}, 19.1 \mathrm{eV}$, is much larger than for $4 \mathrm{~d}$.

The $4 \mathrm{~d}$ XPS for open shell $\mathrm{Ce}^{3+}$ is quite different, Fig. 2(c). The XPS intensity is spread over $\sim 12 \mathrm{eV}$, there are many unresolved core-hole states, and the peak envelope shows four distinct features. In particular, the number of unresolved states is indicated in Fig. 2(c) by the number of light curves for the most intense final states of different energies. The low BE feature centered at $\mathrm{E}_{\text {rel }} \approx 0.6 \mathrm{eV}$ is composed of unresolved contributions from three ionic states at $\mathrm{E}_{\text {rel }}=0$. 0.57, and $1.71 \mathrm{eV}$ which arise dominantly from the spin-orbit split ${ }^{3} \mathrm{P}$ coupling of the $4 \mathrm{~d}$ hole and the $4 \mathrm{f}$ electron with small contributions from other multiplets. The different ${ }^{3} \mathrm{P}$ levels are identified in the figure. This feature corresponds to the peak that Mullins et al. [18] associated with $\mathrm{Ce}^{3+}$. Indeed, we will show that low BE satellites of main XPS peaks due to multiplet coupling of valence open shell electrons with a core hole are a general feature for heavy atoms. 
The $3 \mathrm{~d}$ XPS for $\mathrm{Ce}^{3+}$ is shown in Fig, 2d. In contrast to closed shell Ce4+ (Fig. 2b), there are numerous multiplets that contribute to the $3 \mathrm{~d}_{5 / 2}$ and $3 \mathrm{~d}_{3 / 2}$ main peaks (Fig. $2 \mathrm{~d}$ ). However, the multiplet splittings are smaller than for $4 \mathrm{~d} \mathrm{Ce}^{3+}$ (Fig. 2c) which broadens the main peaks without manifesting strong resolved features, although there are weak satellites at $\sim 5 \mathrm{eV}$ higher BE than the main peaks. The calculated multiplet splitting is greater for the $4 \mathrm{~d}$ compared to the $3 \mathrm{~d}$ XPS because the exchange integral is larger for the $4 \mathrm{~d}-4 \mathrm{f}$ compared to the $3 \mathrm{~d}-4 \mathrm{f}$ exchange interaction. For Hartree-Fock $\mathrm{Ce}^{3+}$ orbitals, the average of the d-f exchange interaction, [22] $\bar{G}$, is $2.13 \mathrm{eV}$ for the $4 \mathrm{~d}-4 \mathrm{f}$ exchange, while it is only $0.61 \mathrm{eV}$ for the $3 \mathrm{~d}-4 \mathrm{f}$ exchange, 3.5 times smaller. Exchange integrals are proportional to the product of the nd and $4 \mathrm{f}$ orbitals and are larger when the orbitals are in the same region of space, as is the case for orbitals that have the same principal quantum number such as the $4 \mathrm{~d}$ and $4 \mathrm{f}$ shells. Since these exchange integrals are, in large part, responsible for the calculated multiplet splittings, it is expected that the multiplets will be better separated and more easily resolved when the ionization is in a shell with the same principle quantum number as the open $4 \mathrm{f}$ shell.

Considering the next highest energy shell with principal quantum number four, the calculated 4p XPS for $\mathrm{Ce}^{3+}$ is shown in Fig. 3. Here, as for the $4 \mathrm{~d}$ XPS of $\mathrm{Ce}^{3+}$ the structure is considerably more complex than a simple spin-orbit split $4 \mathrm{p}_{3 / 2}-4 \mathrm{p}_{1 / 2}$ doublet. The $4 \mathrm{p}_{3 / 2}$ peak has four resolved features including a leading low BE shoulder. In contrast, the $4 \mathrm{p}_{1 / 2}$ peak is split into a well resolved doublet separated by $\sim 3.5 \mathrm{eV}$.

The calculated $5 \mathrm{~d}$ XPS for $\mathrm{U}^{6+}\left(5 \mathrm{f}^{0}\right), \mathrm{U}^{5+}\left(5 \mathrm{f}^{1}\right)$, and $\mathrm{U}^{4+}\left(5 \mathrm{f}^{2}\right)$ are shown in Figs. 4(a)-4(c). As expected, the XPS for closed shell $\mathrm{U}^{6+}$ is a simple doublet from the spin-orbit splitting of $5 \mathrm{~d}_{3 / 2}$ 
and $5 \mathrm{~d}_{5 / 2}$. On the other hand, the XPS of both $\mathrm{U}^{5+}\left(5 \mathrm{f}^{1}\right)$ and $\mathrm{U}^{4+}\left(5 \mathrm{f}^{2}\right)$ are complex with resolved structures including a lowest BE peak with modest intensity. For $\mathrm{U}^{5+}$, this peak arises from two levels that are dominantly spin-orbit split ${ }^{3} \mathrm{P}_{0}$ and ${ }^{3} \mathrm{P}_{1}$ multiplets. For $\mathrm{U}^{4+}$, the peak arises from two levels, at $\mathrm{E}_{\mathrm{rel}}=0$ and at $\mathrm{E}_{\mathrm{rel}}=0.1 \mathrm{eV}$. For $\mathrm{U}^{4+}$, which has $25 \mathrm{f}$ open shell electrons, the angular momentum coupling is more complex since the $5 \mathrm{f}^{2}$ open shell couples dominantly to a ${ }^{3} \mathrm{H} \mathrm{RS}$ multiplet which has 33 degenerate states. Still the low BE peaks are dominated by the high spin coupling of the ${ }^{2} \mathrm{D} 5 \mathrm{~d}^{9}$ core shell with the ${ }^{3} \mathrm{H}$ coupled $5 \mathrm{f}^{2}$. The level at $\mathrm{E}_{\text {rel }}=0$ is $94 \%$ a ${ }^{4} \mathrm{~F}$ multiplet while the level at $\mathrm{E}_{\text {rel }}=0.1 \mathrm{eV}$ is $66 \%{ }^{4} \mathrm{~F}$ with strong contamination from other $\mathrm{RS}$ multiplets. While the low BE peak distinguishes $\mathrm{U}^{5+}$ and $\mathrm{U}^{4+}$ from closed shell $\mathrm{U}^{6+}$, the peak may not be as useful to distinguish these two oxidation states of $U$ from each other. However, there are other features of the $5 \mathrm{~d}$ XPS that may well serve to distinguish them. The $5 \mathrm{~d}_{5 / 2}$ feature for $\mathrm{U}^{4+}$ has a smaller FWHM than that for $\mathrm{U}^{5+}$; furthermore the main $5 \mathrm{~d}_{5 / 2}$ is closer to the low BE shoulder for $\mathrm{U}^{4+}$ than it is for $\mathrm{U}^{5+}$. The doublet for the $5 \mathrm{~d}_{3 / 2}$ feature is clearly different between $\mathrm{U}^{4+}$ and $\mathrm{U}^{5+}$. Thus, it is possible that these features could be used to distinguish $\mathrm{U}^{4+}$ from $\mathrm{U}^{5+}$ and this possibility should be examined experimentally.

Inspection of the calculated $5 \mathrm{p}_{3 / 2}$ XPS for $\mathrm{U}^{5+}$ and $\mathrm{U}^{4+}$ indicate both similarities but also distinct differences, Figs. 5(a) and (b). As in all previous cases, there is a moderately intense low BE peak that is dominated by a high spin, low orbital angular momentum coupled multiplet which here is due to the coupling of the $5 \mathrm{p}$ hole with the $5 \mathrm{f}$ shell. The $5 \mathrm{p}_{1 / 2}$ XPS of the U cations are doublets with a splitting of 2-3 eV (not shown). Furthermore, following arguments similar to those used above for the 5d XPS, it may be possible to use also use the 5p XPS to distinguish $\mathrm{U}^{4+}$ and $\mathrm{U}^{5+}$ oxidation states. 
Next, we present a decomposition of the spin-orbit coupled levels for the $4 p$ XPS of $\mathrm{Ce}^{3+}$ and the $5 \mathrm{p}_{3 / 2}$ XPS of $\mathrm{U}^{5+}$ and $\mathrm{U}^{4+}$ into contributions from $\mathrm{RS}$ multiplets in order to confirm the character of the low BE feature that distinguishes open from closed valence shell cations. It will also show how intermediate coupling and multiplet splittings lead to broad complex structures that, in principle, could be resolved with laboratory XPS and higher resolution synchrotron radiation. We consider only the final levels that have an intensity $\geq 10 \%$ of the final level with the largest $I_{\text {rel }}$, which is normalized to $I_{r e l}=1$. For $\mathrm{Ce}^{3+}$, the initial configuration is $4 \mathrm{f}^{1}$ coupled to a ${ }^{2} \mathrm{~F}_{5 / 2}$ level. The final configuration is $4 \mathrm{p}^{5} 4 \mathrm{f}^{1}$ which can couple to the following spin-orbit split singlet and triplet multiplets: ${ }^{3} \mathrm{D}_{1,2,3},{ }^{3} \mathrm{~F}_{2,3,4},{ }^{3} \mathrm{G}_{3,4,5}$, and ${ }^{1} \mathrm{D}_{2},{ }^{1} \mathrm{~F}_{3},{ }^{1} \mathrm{G}_{4}$. [22] XPS excitations are allowed to all these multiplets although the selection rules do not ionization to the $\mathrm{J}=5$ coupling of the ${ }^{3} \mathrm{G}$ multiplet; see Ref. [11] and references, therein. In Table I, information about the levels for the $4 p$ XPS of $\mathrm{Ce}^{3+}$ is presented; this table should be examined in connection with Fig. 3 where the $4 p$ XPS is plotted. The following information is given for each level in: (1) The energy of the level. $E_{\text {rel }}$ with respect to the lowest $4 p^{5} 4 f^{1}$ level at $E_{\text {rel }}=0$. (2) The $I_{\text {rel }}$ with the $I_{\text {rel }}$ normalized to one for the XPS most intense level. (3) The J value for the level which is, for the atoms, the only good quantum number for angular momentum and will contain a mixture of RS multiplets. And, (4) the decomposition of the levels into their composition in terms of RS multiplets given as percentages. This composition is determined by projecting non-relativistic wavefunctions for the multiplets on the relativistic wavefunctions for the levels. [25] Because of the degeneracy of the ${ }^{3} \mathrm{~F}$ and ${ }^{1} \mathrm{~F}$ multiplets, it is not always possible for us to separate the ${ }^{3} \mathrm{~F}$ and ${ }^{1} \mathrm{~F}$ contributions to the levels and these multiplets are grouped together in Table I. (For the energies of multiplets, see Ref. [38] and references therein for formal expressions and Ref. [39] for a program to calculate these expressions.) The two lowest levels at $\mathrm{E}_{\mathrm{rel}}=0$ and $1.3 \mathrm{eV}$, which, as shown by the underlying contributions in Fig. 3, form the low BE shoulder in the 4p XPS are 
dominantly ${ }^{3} \mathrm{D}$ multiplets. The lowest level is a pure ${ }^{3} \mathrm{D}$ since only the ${ }^{3} \mathrm{D}$ multiplet can couple to a $\mathrm{J}=1$ level. The second level at $\mathrm{E}_{\mathrm{rel}}=1.3 \mathrm{eV}$ is $85 \%{ }^{3} \mathrm{D}$ with other multiplets contributing the remaining $15 \%$. In other words, a high spin coupled multiplet between the $4 \mathrm{p}^{5}$ and $4 \mathrm{f}^{1}$ shells forms the low BE shoulder in the $4 p$ XPS of $\mathrm{Ce}^{3+}$; of course, such multiplet satellites are not possible for closed shell $\mathrm{Ce}^{4+}$. We have also identified high spin multiplets a leading to distinct and observable low BE features for the $4 \mathrm{~d}$ XPS of $\mathrm{Ce}^{3+}$ and for the $5 \mathrm{~d}$ XPS of $\mathrm{U}^{5+}$ and $\mathrm{U}^{4+}$. The other levels for the $4 \mathrm{p}$ XPS of $\mathrm{Ce}^{3+}$ are strong mixtures of different RS multiplets; in no case does the contribution of a single multiplet exceed $66 \%$. This shows the importance of intermediate coupling for the core configurations of heavy atoms; this is a general feature even for core level excitations in light atoms. [40] Similar information is presented in Tables II and III for the $5 \mathrm{p}_{3 / 2}$ XPS of $\mathrm{U}^{5+}$ and $\mathrm{U}^{4+}$ respectively. The same RS multiplets and levels arise for $\mathrm{U}^{5+}$ because there are the same open shell occupations as for $\mathrm{Ce}^{3+}$, albeit with different principle quantum numbers. Indeed, the analyses of the $4 p$ XPS structure of $\mathrm{Ce}^{3+}$ and the $5 p$ XPS of $\mathrm{U}^{5+}$, compare Tables I and II and Figs. 3 and 5(a), are quite similar. In particular, the low BE feature is dominated by the level at $\mathrm{E}_{\text {rel }}=0$ is, for $\mathrm{U}^{5+}$, still $100 \%$ a ${ }^{3} \mathrm{D}$ multiplet and the second level at $\mathrm{E}_{\mathrm{rel}}-1.4 \mathrm{eV}$ is a $\mathrm{J}=2$ level that is dominantly ${ }^{3} \mathrm{D}$, albeit with a larger contamination from the ${ }^{3} \mathrm{~F}$ multiplet. For the other levels, the mixings of different RS multiplets is somewhat larger than for $\mathrm{Ce}^{3+}$, which is not at all surprising given the larger spin-orbit splittings for the U $5 p$ shell compared to the Ce $4 p$ shell. For $\mathrm{U}^{4+}$ with a $5 \mathrm{f}^{2}$ configuration, the initial state is a $\mathrm{J}=4$ level which is dominantly, $91 \%$, a ${ }^{4} \mathrm{H}$ multiplet. [25] For a ${ }^{4} \mathrm{H}$ multiplet, the 5p XPS allowed ionic states [11] are doublet and quartets with orbital angular momentum $\mathrm{G}(\mathrm{L}=4), \mathrm{H}(\mathrm{L}=5)$, and $\mathrm{I}(\mathrm{L}=6)$. For a $\mathrm{J}=4$ level, the selection rules for allowed XPS final states is that they must have $J=11 / 2,9 / 2$. $7 / 2$, or $5 / 2$. In Table III, we present properties of the $5 \mathrm{p}_{3 / 2}$ XPS final levels with $\mathrm{I}_{\mathrm{rel}} \geq 0.1$ analogous to those in Tables I and II. In order to be compact, we restrict ourselves to providing details of the decomposition of the 
levels in terms of their composition with high spin, quartet multiplets since this is sufficient to show that the two main points established earlier also apply to $\mathrm{U}^{4+}$. The first two $5 \mathrm{p}_{3 / 2}$ hole levels at $\mathrm{E}_{\mathrm{rel}}=0$ and $0.8 \mathrm{eV}$, which contribute to the clear low BE feature in the $5 \mathrm{p}_{3 / 2}$ XPS, see fig. 5(b), are dominated by the high spin, ${ }^{4} \mathrm{G}$, multiplet, although they have a larger contamination from other multiplets. Except for the low $\mathrm{I}_{\text {rel }}$ level at $\mathrm{E}_{\mathrm{rel}}=2.2 \mathrm{eV}$, the other levels have significant contributions from quartet multiplets but also, not shown, from doublet multiplets; this again establishes the strong intermediate coupling of these levels. The evidence we have presented for Ce and $\mathrm{U}$ suggests that a low BE feature that is high spin coupled may be a general feature of the XPS of open shell $\mathrm{f}$ systems. Furthermore, it is possible that the differences of the XPS multiplet splittings for different charge states of the metal cation could be observable if the XPS measurements have adequate resolution.

This section closes with a comment on the potential of solid state effects to modify and change the atomic multiplet splittings discussed at length above. In particular, the concern is for the modifications that might be induced by ligand field effects. In order to treat these effects, the embedded cluster models of $\mathrm{XO}_{8}$ shown in Fig. 1 are used to model $\mathrm{UO}_{2}$ with a nominal oxidation state of IV and $\mathrm{CeO}_{2}$ with a nominal oxidation state of III. Although, the actual occupations of the $\mathrm{Ce}(4 \mathrm{f})$ and $\mathrm{U}(5 \mathrm{f})$ shells may be different from the nominal oxidation states because of covalent mixings of these orbitals with $\mathrm{O}(2 \mathrm{p})$, [41] the oxidation states correctly reflect the integral occupations of the open shells, 1 for $\mathrm{Ce}(\mathrm{III})$ and 2 for $\mathrm{U}(\mathrm{IV})$. The $\mathrm{CeO}_{2}$ crystal structure used is, of course, not that of $\mathrm{Ce}_{2} \mathrm{O}_{3}$, but there is evidence that reduced $\mathrm{Ce}(\mathrm{III})$ in Ceria occupies local fluorite sites. [42] The ligand field induces splittings of the levels of the isolated cation into multiplets appropriate for the cubic double group; [43] it also may change the splittings of the different levels because the covalent character present in the compound modifies 
the exchange interactions, specifically the exchange integrals, that split the multiplets. [12] For example, the 9 fold degenerate lowest ${ }^{3} \mathrm{H}_{4}$ level of $\mathrm{U}^{4+}$ is split into a several levels in the cubic environment of $\mathrm{UO}_{2}$. The lowest of these levels is a 3 fold degenerate $\Gamma_{5}$ multiplet with the next excited state lying at $0.15 \mathrm{eV}$ higher in energy. [44]

While the changes in the degeneracies and the splittings of the ground and coreionized states of the compound compared to the isolated cation do lead to changes in the precise energies and intensities of the final levels, they do not lead to major or even easily observable changes in the XPS. To demonstrate this, we compare, in Fig. 6, the U $5 \mathrm{~d}$ XPS for $\mathrm{UO}_{2}$, including ligand field effects with the U 5d XPS for the isolated $\mathrm{U}^{4+}$ cation, where both are plotted over the same energy range. There are differences but they are minor and consistent with smaller multiplet splittings in the cluster model because of a covalent mixing of the metal and ligand orbitals. XPS spectra for the $U\left(5 p_{3 / 2}\right)$ and for the Ce $4 p$ and $4 d$, not shown, are also very similar between the cluster models and the isolated cations. In other words, the atomic effects are preserved in the presence of ligand field splittings and our analysis of the low BE high-spin multiplet features for the isolated cations applies to condensed systems. Because these atomic effects dominate, it is reasonable to conclude that it may be possible to distinguish different open shell occupations from each other as well as to distinguish open shell from closed shell configurations. It is possible to distinguish the different open shell occupations due to additional features in the XPS besides the low BE feature, which is a fingerprint of the presence of open shells. Our discussion above strongly suggests that the features of the atomic U $5 \mathrm{~d}$ and $5 \mathrm{p}$ XPS, shown in Figs. 4 and 5, will be preserved even when ligand field effects are taken in to account. 
The many body effects involving shake, or charge transfer, [11] have not been taken into account in our many body treatment for the cluster models. While these effects are especially important for the $3 \mathrm{~d}$ and $4 \mathrm{~d}$ XPS of Ce(IV) and for the $3 \mathrm{~d}$ XPS of Ce(III), $[18,31]$ comparison of our predictions with experiment, made in the next section, will show that they are less important for the $4 \mathrm{~d}$ XPS of $\mathrm{Ce}(\mathrm{III})$. In this regard, we will provide an explanation of why an atomic model may be better for $\mathrm{Ce}(\mathrm{III})$ than $\mathrm{Ce}(\mathrm{IV})$. In any event, it is clear from the discussion above that the multiplet splittings analyzed in detail may lead to observable XPS features; this is further demonstrated by comparison with experiment.

\section{Observable Multiplets in Ce and U XPS: Comparison With Experiment}

In Fig. 7, we show, side by side, our predictions for the $4 \mathrm{~d}$ XPS of $\mathrm{Ce}^{3+}$ with the experimental data taken from the results of Mullins et al. [45] for $\mathrm{Ce}_{2} \mathrm{O}_{3}$. The main features of our theoretical spectrum are labelled A-D. We have also labelled the low BE features in the $\mathrm{Ce}_{2} \mathrm{O}_{3}$ spectra [45] as $\mathrm{D}$ to show that we associate the experimental feature with this low $\mathrm{BE}$, high spin, dominantly ${ }^{3} \mathrm{P}$, multiplet of the $4 \mathrm{~d}^{9} 4 \mathrm{f}^{1}$ configuration of $\mathrm{Ce}^{3+}$. Furthermore, the general character of the features A-C are present in the experiment. The calculated B and C peaks are at roughly the same energy relative to D and have roughly the same separation as the experimental features at 108 and $112 \mathrm{eV}$. Furthermore, the shoulder at $116 \mathrm{eV}$ can be associated with feature A. The relative intensities of the features $\mathrm{B}$ and $\mathrm{C}$ are reversed from the measurement but this could arise from factors that we have not taken into account including differential loss of intensity to the satellites at $\sim 125 \mathrm{eV}$. These satellites are not present in our atomic spectra since they arise from excitations that depend on the crystalline environment. It is important to note however, that these satellites are much less intense than for the $4 \mathrm{~d}$ XPS of $\mathrm{Ce}$ (III) than $\mathrm{Ce}(\mathrm{IV})$. 
$[18,45]$ We suggest that this may be related to a reduced covalent mixing of $\mathrm{Ce}(4 \mathrm{f})$ and $\mathrm{Ce}(5 \mathrm{~d})$ with $\mathrm{O}(2 \mathrm{p})$, for $\mathrm{Ce}(\mathrm{III})$ than for $\mathrm{Ce}(\mathrm{IV})$ since this covalent mixing is a major driving for giving intensity to shake XPS satellites, [11, 19]. Preliminary results for an analysis of the $4 \mathrm{f}$ occupations in the closed shell orbitals of the $\mathrm{CeO}_{8}$ cluster models for both $\mathrm{Ce}(\mathrm{IV})$ and $\mathrm{Ce}(\mathrm{III})$ show a significant reduction of $\mathrm{Ce}(4 \mathrm{f})$ character in the closed shells, which is consistent with a reduced shake satellite intensity. In Fig. 8, we present a comparison of our theoretical predictions for the $4 \mathrm{~d}$ XPS of $\mathrm{Ce}^{3+}$ with our measured XPS for a $\mathrm{U}$ doped sample of $\mathrm{CeO}_{2}$ that is $\mathrm{Ce}_{0.95} \mathrm{U}_{0.05} \mathrm{O}_{2}$ where the doped sample has been sputtered for 5 minutes to increase the amount of reduced $\mathrm{Ce}(\mathrm{III})$, especially in the depth sampled by the XPS. Here, we have subtracted a Tougaard background [46-47] from the experimental data. The lowest $\mathrm{BE}$ feature at $\mathrm{E}_{\mathrm{rel}} \approx-7 \mathrm{eV}$, or $\mathrm{E} \approx 96 \mathrm{eV}$, is associated with the $\mathrm{U} 5 \mathrm{~d}_{5 / 2}$ peak arising from the $\mathrm{U}$ doping. It is possible that the $\mathrm{U} 5 \mathrm{~d}_{3 / 2}$ will make some contribution to the low BE feature $\mathrm{D}$, for the separation of $\mathrm{U} 5 \mathrm{~d}_{5 / 2}$ and $\mathrm{U}$ $5 d_{3 / 2}$ peaks see Fig. 4. However, as seen in Fig. 4, this contribution will be considerably smaller than that for the $5 d_{5 / 2}$ and we expect the feature $\mathrm{D}$ to be dominated by the high spin multiplet of the $4 \mathrm{~d}-4 \mathrm{f}$ coupling in $\mathrm{Ce}(\mathrm{III})$. The $\mathrm{U}$ doped $\mathrm{CeO}_{2}$ spectra is similar to the $\mathrm{Ce}_{2} \mathrm{O}_{3} 4 \mathrm{~d}$ XPS in Fig. 7 strongly indicating that $\mathrm{Ce}^{3+}$ is dominantly present in the doped sample. The shake satellites at $\mathrm{E}_{\mathrm{rel}} \approx 20 \mathrm{eV}$ are more intense than in Fig. 7 for $\mathrm{Ce}_{2} \mathrm{O}_{3}$ but they are well separated from the features A-D which are characterisitic of $\mathrm{Ce}(\mathrm{III})$.

In Fig. 9, we compare the calculated U 5p $3 / 2$ XPS for the embedded U(IV) $\mathrm{O}_{8}$ cluster with our experimental measurements for $\mathrm{UO}_{2}$. The $\mathrm{UO}_{2}$ sample was freshly cleaved and a Tougaard background [46-47] has been subtracted. The main doublet of the theory is present in the measurement although the experimental features are considerably broadened. The satellites at $\mathrm{E}_{\text {rel }}$ $\sim 4$ and $\sim 6 \mathrm{eV}$ also appear to be present in the measured spectra. As shown in Table III,for the $\mathrm{U}^{4+}$ 
$5 \mathrm{p}_{3 / 2} \mathrm{XPS}$, the low BE feature is dominated by spin-orbit split components of the high spin ${ }^{4} \mathrm{G}$ multiplet, which arises from the coupling of the open $5 p$ and $5 f$ shells. On the other hand, the $\sim 6$ $\mathrm{eV}$ satellites arise from other multiplets.

\section{Conclusions and Summary}

We have presented detailed evidence that multiplet splittings of the XPS of suitably chosen core regions can provide a means of characterizing the oxidation state, or open shell occupation, of heavy metal cations, especially in ionic compounds. A key for being able to use XPS multiplet splittings to distinguish oxidation states is to select a core region where the exchange integrals between the core-ionized shell and the open valence shell, $4 \mathrm{f}$ for lanthanides and $5 \mathrm{f}$ for actinides are large. This means choosing a core-level with the same principle quantum number as the open valence shell since these core orbitals are large in the same region of space as the orbitals of the open valence shell. The intermediate coupling for the heavy atoms, where spinorbit coupling is important even for the outer shells, leads to multiplets distributed over several $\mathrm{eV}$ and often with several distinguishable features. However, in all the cases we have examined, there is a low BE multiplet with reasonable XPS intensity that can serve as a fingerprint that distinguishes open shell systems from closed shell systems where multiplet splitting in the XPS is not present. In particular, we have shown that this low BE peak arises from a high spin coupled multiplet, which helps to insure that the multiplet receives reasonable XPS intensity. Our work comparing $\mathrm{U}^{5+}$ and $\mathrm{U}^{4+}$ also suggests that it might be possible to use these low $\mathrm{BE}$ multiplets to distinguish oxidation states with different numbers of open shell electrons. 
The use of multiplet splittings to characterize oxidation states is a method that has been widely applied for transition metal compounds. Our results provide evidence that these splittings can also be used to study heavy metals. A difficulty is that the core shells that have the largest multiplet splittings may not have large XPS intensity; certainly, they do not have the largest XPS intensity. However, especially given the availability of synchrotron radiation, this may not be a serious limitation. In any case, our comparisons of theory with experiment for both the Ce $4 d$ XPS and the U 5p XPS show that the multiplet splittings can be resolved with laboratory XPS. Thus, our work indicates that there may be a new tool to characterize the oxidation states of heavy metal cation that can be used to supplement existing methods.

\section{Acknowledgements.}

PSB and ESI acknowledge support from the U.S. Department of Energy, Office of

Science, Office of Basic Energy Sciences, Chemical Sciences, Geosciences, and Biosciences (CSGB) Division through the Geosciences program at Pacific Northwest National Laboratory. YS and HI thank Dr. Toseef Ahmed for his assistance in the XPS measurements.

\section{References}

1. R. P. Gupta and S. K. Sen, Phys. Rev. B 10, 71 (1974).

2. R. P. Gupta and S. K. Sen, Phys. Rev. B 12, 15 (1975).

3. C. S. Fadley, et al., Phys. Rev. Lett. 23, 1397 (1969).

4. P. S. Bagus, A. J. Freeman, and F. Sasaki, Phys. Rev. Lett. 30, 850 (1973). 
5. A. J. Freeman, P. S. Bagus, and J. V. Mallow, Int. J. Mag. 4, 49 (1973).

6. E.-K. Viinikka and Y. Öhrn, Phys. Rev. B 11, 4168 (1975).

7. B. D. Hermsmeier, et al., Phys. Rev. B 48, 12425 (1993).

8. P. S. Bagus, E. S. Ilton, and J. R. Rustad, Phys. Rev. B 69, 205112 (2004).

9. E. S. Ilton, W. A. de Jong, and P. S. Bagus, Phys. Rev. B 68, 125106 (2003).

10. P. S. Bagus, R. Broer, and E. S. Ilton, J. Electron Spectrosc. Relat. Phenom. 165, 46 (2008).

11. P. S. Bagus, E. S. Ilton, and C. J. Nelin, Surf. Sci. Rep. 68, 273 (2013).

12. P. S. Bagus and E. S. Ilton, Phys. Rev. B 73, 155110 (2006).

13. P. S. Bagus and E. S. Ilton, Theor. Chem. Accounts 118, 495 (2007).

14. V. Tsurkan, et al., Solid State Commun. 114, 149 (2000).

15. P. S. Bagus, R. Broer, and E. S. Ilton, Chem. Phys. Lett. 394, 150 (2004).

16. P. S. Bagus and J. V. Mallow, Chem. Phys. Lett. 228, 695 (1994).

17. E. S. Ilton and P. S. Bagus, Surf. Interface Anal. 43, 1549 (2011).

18. D. R. Mullins, S. H. Overbury, and D. R. Huntley, Surf. Sci. 409, 307 (1998).

19. P. S. Bagus, C. J. Nelin, and E. S. Ilton, J. Chem. Phys. 139, 244704 (2013).

20. A. Kotani and H. Ogasawara, Physica B 186-188, 16 (1993).

21. E. S. Ilton and P. S. Bagus, Phys. Rev. B 71, 195121 (2005).

22. J. C. Slater, Quantum Theory of Atomic Structure, Vols. I \& II (McGraw-Hill, New York, 1960).

23. L. Visscher, et al., Comput. Phys. Commun. 81, 120 (1994).

24. W. A. de Jong, L. Visscher, and W. C. Nieuwpoort, THEOCHEM 458, 41 (1999).

25. P. S. Bagus, et al., Chem. Phys. Lett. 546, 58 (2012). 
26. T. Aberg, Phys. Rev. 156, 35 (1967).

27. P. S. Bagus, et al., Phys. Rev. A 9, 1090 (1974).

28. J. A. Gubner, J. Phys. A 27, L745 (1994).

29. R. W. G. Wyckoff, Crystal Structures (Wiley, New York, 1963).

30. J. Paier, C. Penschke, and J. Sauer, Chemical Reviews 113, 3949 (2013).

31. C. J. Nelin, et al., Int. J. Quantum Chem. 110, 2752 (2010).

32. DIRAC, a relativistic ab initio electronic structure program, Release DIRACO8 (2008), written by L. Visscher, H. J. Aa. Jensen, and T. Saue, with new contributions from R. Bast, S. Dubillard, K. G. Dyall, U. Ekström, E. Eliav, T. Fleig, A. S. P. Gomes, T. U. Helgaker, J. Henriksson, M. Iliaš, Ch. R. Jacob, S. Knecht, P. Norman, J. Olsen, M. Pernpointner, K. Ruud, P. Sałek, and J. Sikkema (see the URL at http://dirac.chem.sdu.dk. .

33. F. Prosser and S. Hagstrom, Int. J. Quantum Chem. 2, 89 (1968).

34. F. Prosser and S. Hagstrom, J. Chem. Phys. 48, 4807 (1968).

35. CLIPS is a program system to compute ab initio SCF and correlated wavefunctions for polyatomic systems. It has been developed based on the publicly available programs in the ALCHEMY package from the IBM San Jose Research Laboratory by P. S. Bagus, B. Liu, A. D. McLean, and M. Yoshimine.

36. Y. Al-Salik, I. Al-Shankiti, and H. Idriss, J. Electron Spectrosc. Relat. Phenom. 194, 66 (2014).

37. A. Kotani, T. Jo, and J. C. Parlebas, Advances in Physics 37, 37 (1988).

38. E. U. Condon and G. H. Shortly, The Theory of Atomic Spectra (Cambridge University Press, Cambridge, 1951).

39. C. F. Fischer, Comput. Phys. Commun. 128, 635 (2000).

40. P. S. Bagus, M. J. Sassi, and K. M. Rosso, J. Electron Spectrosc. Relat. Phenom. (in press).

41. P. S. Bagus and C. J. Nelin, J. Electron Spectrosc. Relat. Phenom. 194, 37 (2014).

42. A. M. Burow, et al., J. Chem. Phys. 130 (2009).

43. J. S. Griffith, The Theory of Transition-Metal lons (Cambridge Press, Cambridge, 1971). 
44. J. G. Tobin, et al., Phys. Rev. B (submitted).

45. D. R. Mullins, P. V. Radulovic, and S. H. Overbury, Surf. Sci. 429, 186 (1999).

46. M. P. Seah, Surf. Sci. 420, 285 (1999).

47. M. P. Seah, I. S. Gilmore, and S. J. Spencer, Surf. Sci. 461, 1 (2000). 
Table I. Properties of the intense 4p XPS ionic levels for $\mathrm{Ce}^{3+}$. For each level, the character, either dominantly $4 p_{3 / 2}$ or $4 p_{1 / 2}$, is given along with the XPS $E_{\text {rel }}$ and $I_{\text {rel }}$ and $J$, the total angular momentum coupling of the level taking spin-orbit coupling into account. In addition, the contributions of the different RS multiplets to the relativistic J level are given, see text.

\begin{tabular}{|l|l|l|l|l|l|l|l|l|}
\hline Character & $\mathrm{E}_{\text {rel-eV }}$ & $\mathrm{I}_{\text {rel }}$ & $\mathrm{J}$ & $\%\left({ }^{3} \mathrm{D}\right)$ & $\%\left({ }^{3} \mathrm{G}\right)$ & $\%\left({ }^{3} \mathrm{~F}\right)+\%\left({ }^{1} \mathrm{~F}\right)$ & $\%\left({ }^{1} \mathrm{D}\right)$ & $\%\left({ }^{1} \mathrm{G}\right)$ \\
\hline $4 \mathrm{p}_{3 / 2}$ & 0 & 0.34 & 1 & 100.0 & --- & --- & --- & --- \\
\hline & 1.27 & 0.37 & 2 & 85.0 & --- & $13.2^{\mathrm{a}}$ & 1.8 & --- \\
\hline & 2.57 & 1.00 & 4 & --- & 63.4 & $5.8^{\mathrm{a}}$ & --- & 30.8 \\
\hline & 2.94 & 0.10 & 3 & 58.1 & 0.2 & 41.7 & --- & --- \\
\hline & 4.26 & 0.69 & 3 & 0.3 & 33.9 & 65.7 & --- & --- \\
\hline & 7.81 & 0.21 & 2 & 2.7 & --- & $46.9^{\mathrm{a}}$ & 50.4 & --- \\
\hline $4 \mathrm{p}_{1 / 2}$ & 21.79 & 0.79 & 3 & 0.3 & 65.4 & 34.3 & --- & --- \\
\hline & 25.38 & 0.55 & 2 & 12.0 & --- & $39.9^{\mathrm{a}}$ & 47.8 & --- \\
\hline
\end{tabular}

${ }^{\mathrm{a}}$ For this state the entire contribution is from a ${ }^{3} \mathrm{~F}$ multiplet.

Table II. Properties of the intense $5 \mathrm{p}_{3 / 2}$ XPS ionic levels for $\mathrm{U}^{5+}$; see caption to Table I and text.

\begin{tabular}{|l|c|l|l|l|l|l|l|l|}
\hline Character & $\mathrm{E}_{\text {rel- }} \mathrm{eV}$ & $\mathrm{I}_{\text {rel }}$ & $\mathrm{J}$ & $\%\left({ }^{3} \mathrm{D}\right)$ & $\%\left({ }^{3} \mathrm{G}\right)$ & $\%\left({ }^{3} \mathrm{~F}\right)+\%\left({ }^{1} \mathrm{~F}\right)$ & $\%\left({ }^{1} \mathrm{D}\right)$ & $\%\left({ }^{1} \mathrm{G}\right)$ \\
\hline $4 \mathrm{p}_{3 / 2}$ & 0 & 0.33 & 1 & 100.0 & --- & --- & --- & --- \\
\hline & 1.38 & 0.41 & 2 & 76.3 & --- & $22.6^{\mathrm{a}}$ & 1.2 & --- \\
\hline & 2.00 & 1.00 & 4 & --- & 57.2 & $5.1^{\mathrm{a}}$ & --- & 38.0 \\
\hline & 3.02 & 0.49 & 3 & 31.4 & 9.5 & 59.4 & --- & -- \\
\hline & 3.64 & 0.29 & 3 & 16.8 & 17.8 & 65.5 & --- & --- \\
\hline & 6.51 & 0.15 & 2 & 4.6 & --- & $33.2^{\mathrm{a}}$ & 62.2 & --- \\
\hline
\end{tabular}

${ }^{a}$ For this state the entire contribution is from a ${ }^{3} \mathrm{~F}$ multiplet. 
Table III. Properties of the intense $5 \mathrm{p}_{3 / 2}$ XPS ionic levels for $\mathrm{U}^{4+}$. The format of this table is similar to that for Table I; see caption to Table I and text.

\begin{tabular}{|l|l|l|l|l|c|c|}
\hline Character & $\mathrm{E}_{\text {rel }}-\mathrm{eV}$ & $\mathrm{I}_{\mathrm{rel}}$ & $\mathrm{J}$ & $\%\left({ }^{4} \mathrm{G}\right)$ & $\%\left({ }^{4} \mathrm{H}\right)$ & $\%\left({ }^{4} \mathrm{I}\right)$ \\
\hline $4 \mathrm{p}_{3 / 2}$ & 0 & 0.49 & $5 / 2$ & 87.3 & --- & --- \\
\hline & 0.80 & 0.34 & $7 / 2$ & 71.7 & 13.8 & --- \\
\hline & 2.23 & 0.12 & $7 / 2$ & 1.5 & 3.5 & --- \\
\hline & 2.35 & 1.00 & $11 / 2$ & 3.5 & 13.0 & 40.9 \\
\hline & 2.64 & 0.10 & $11 / 2$ & 66.1 & 11.2 & 0.3 \\
\hline & 2.82 & 0.71 & $9 / 2$ & 1.4 & 24.0 & 14.3 \\
\hline & 4.42 & 0.18 & $7 / 2$ & 21.4 & 13.7 & --- \\
\hline & 6.65 & 0.12 & $9 / 2$ & 9.6 & 1.5 & 6.8 \\
\hline
\end{tabular}


Figure Captions

Figure 1. Schematic view of the embedded $\mathrm{XO}_{8}$ cluster to model $\mathrm{CeO}_{2}$ and $\mathrm{UO}_{2}$ where the central atom is the metal surrounded by its 8 nearest neighbor $\mathrm{O}$ anions and by a representative set of point charges shown as small circles. The colors, shown online, are as follows: The central cation is blue; the eight $\mathrm{O}$ anions are green, and the point charges are red for -2 and blue for $+4 /$

Figure 2. Plots of the Ce XPS with a $1.5 \mathrm{eV}$ FWHM Gaussian broadening and with the lowest energy core-hole state at $\mathrm{E}_{\mathrm{rel}}=0.0 \mathrm{eV}$ : (a) $4 \mathrm{~d}$ XPS of $\mathrm{Ce}^{4+}$; (b) $3 \mathrm{~d}$ XPS of $\mathrm{Ce}^{4+}$; (c) $4 \mathrm{~d}$ XPS of $\mathrm{Ce}^{3+}$; and (d) $3 \mathrm{~d}$ XPS of $\mathrm{Ce}^{3+}$. In (c), the different ${ }^{3} \mathrm{P}_{0},{ }^{3} \mathrm{P}_{1}$, and ${ }^{3} \mathrm{P}_{2}$ levels that contribute to the low BE XPS peak are identified.

Figure 3. Plot of the 4p XPS of $\mathrm{Ce}^{3+}$; see caption to Fig. 2.

Figure 4. Plot of the 5d XPS for: (a) $\mathrm{U}^{6+}$, (b) $\mathrm{U}^{5+}$, and (c) $\mathrm{U}^{4+}$. See caption to Fig. 2.

Figure 5. Plots of the $5 p_{3 / 2}$ XPS for: (a) $U^{5+}$ and (b) $U^{4+}$. See caption to Fig. 2

Figure 6. Comparison of the 5d XPS for: (a) $\mathrm{U}^{4+}$ and (b) $\mathrm{UO}_{2}$; see caption to Fig. 2.

Figure 7. Comparison of the calculated $4 \mathrm{~d}$ XPS of $\mathrm{Ce}^{3+}$ with data for the measured $4 \mathrm{~d}$ XPS for $\mathrm{Ce}_{2} \mathrm{O}_{3}$ taken from Ref. [45]. The features of the theoretical spectra are labelled A-D and the label $\mathrm{D}$ is given to the low. $105 \mathrm{eV}, \mathrm{BE}$ feature of the experiment. See caption to Fig. 2.

Figure 8. Comparison of the calculated 4d XPS of $\mathrm{Ce}^{3+}$ with the measured $4 \mathrm{~d}$ XPS for U doped and sputtered $\mathrm{CeO}_{2}$; see text. The peaks are labelled as in Fig. 7. The theoretical peak B has been aligned with the maxima of the XPS for doped $\mathrm{CeO}_{2}$. See caption to Fig. 2.

Figure 9. Comparison of the theoretical U 5 $\mathrm{p}_{3 / 2} \mathrm{XPS}$ for the $\mathrm{UO}_{8}$ cluster model of $\mathrm{UO}_{2}$ with the measured $5 p_{3 / 2}$ XPS for $\mathrm{UO}_{2}$; see caption to Fig. 2. The maxima of the main experimental and theoretical peaks are aligned. 


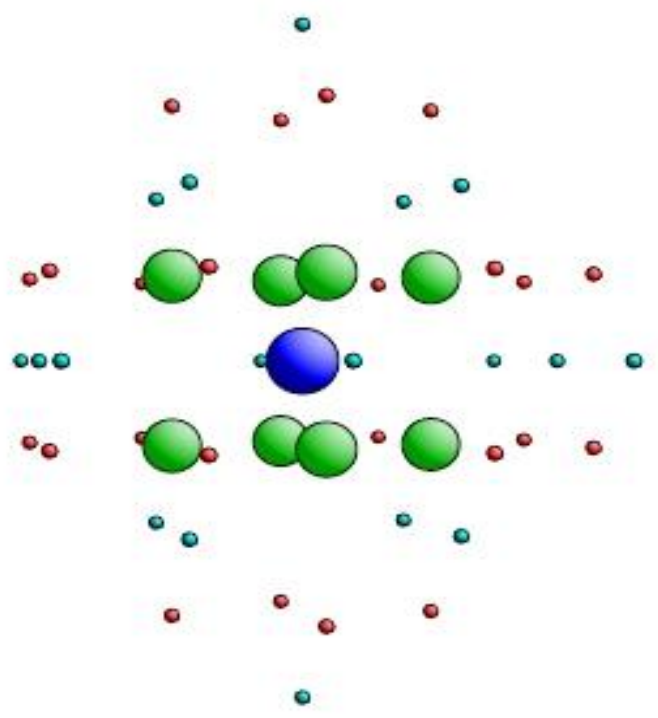

Figure 1. Schematic view of the embedded $\mathrm{XO}_{8}$ cluster to model $\mathrm{CeO}_{2}$ and $\mathrm{UO}_{2}$ where the central atom is the metal surrounded by its 8 nearest neighbor $\mathrm{O}$ anions and by a representative set of point charges shown as small circles. The colors, shown online, are as follows: The central cation is blue; the eight $\mathrm{O}$ anions are green, and the point charges are red for -2 and blue for $+4 /$
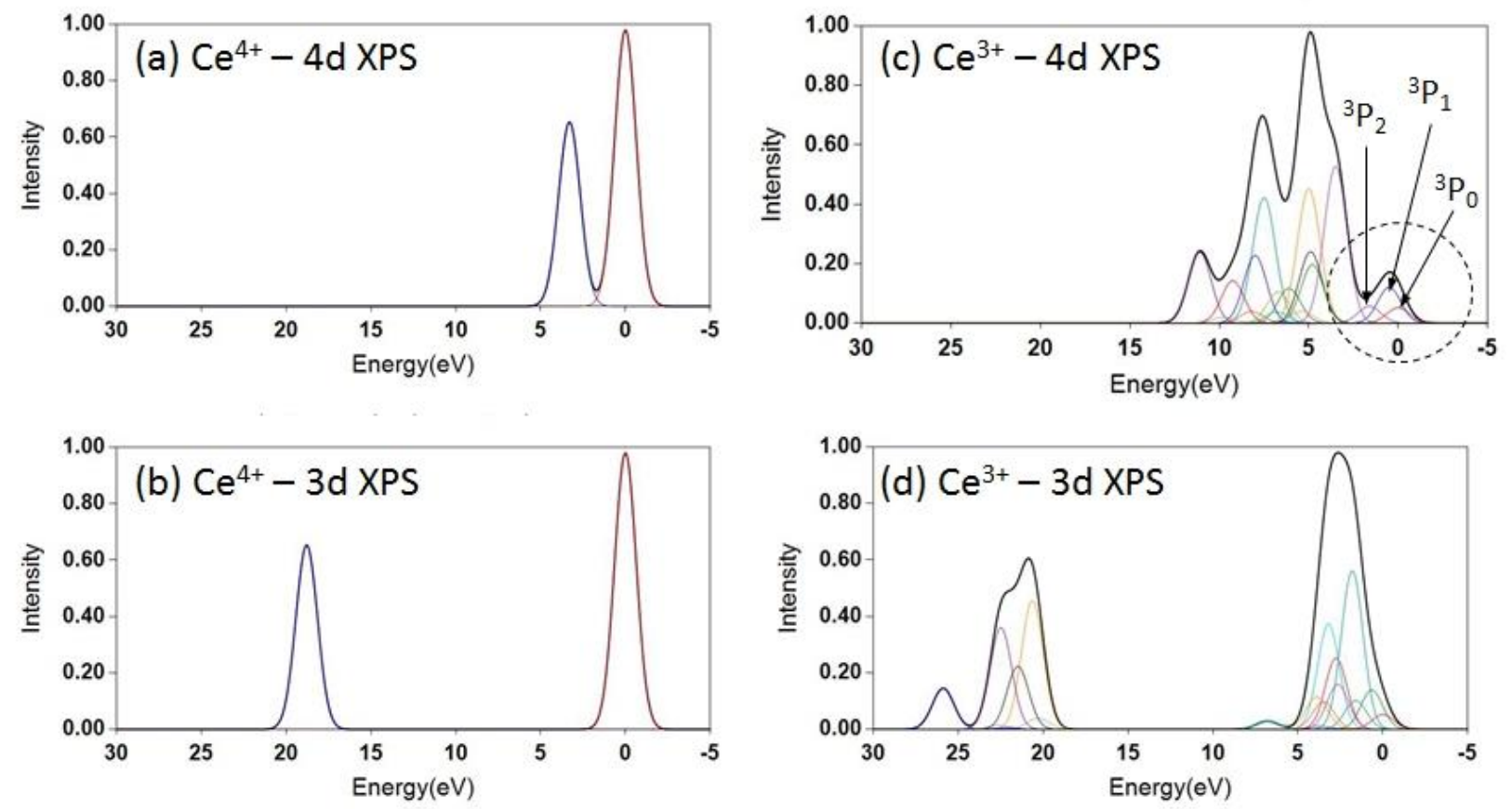

Figure 2. Plots of the Ce XPS with a 1.5 eV FWHM Gaussian broadening and with the lowest energy core-hole state at $\mathrm{E}_{\mathrm{rel}}=0.0 \mathrm{eV}$ : (a) $4 \mathrm{~d}$ XPS of $\mathrm{Ce}^{4+}$; (b) $3 \mathrm{~d}$ XPS of $\mathrm{Ce}^{4+}$; (c) $4 \mathrm{~d}$ XPS of $\mathrm{Ce}^{3+}$; and (d) $3 \mathrm{~d}$ XPS of $\mathrm{Ce}^{3+}$. In (c), the different ${ }^{3} \mathrm{P}_{0},{ }^{3} \mathrm{P}_{1}$, and ${ }^{3} \mathrm{P}_{2}$ levels that contribute to the low BE XPS peak are identified. 


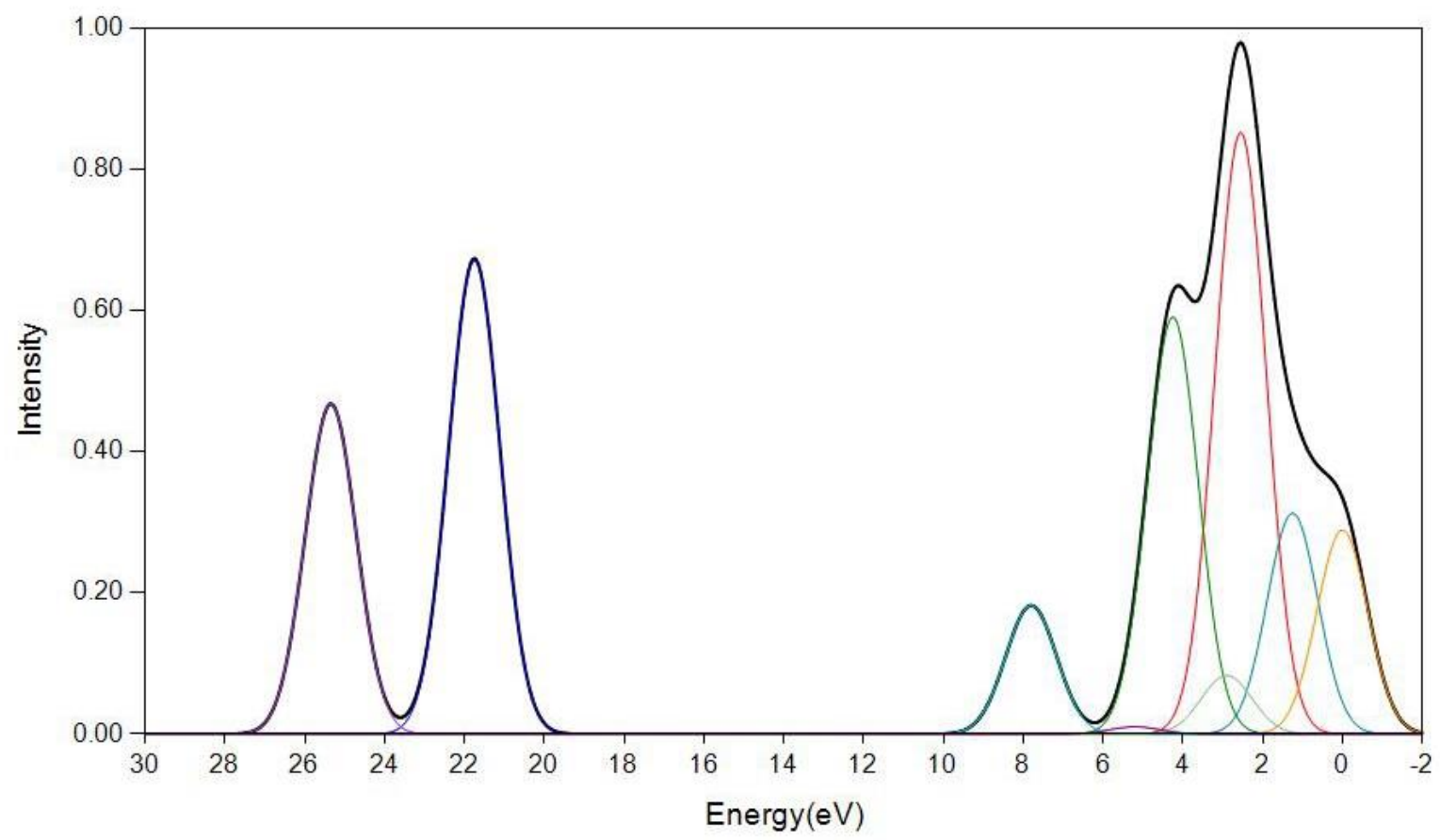

Figure 3. Plot of the $4 p$ XPS of $\mathrm{Ce}^{3+}$; see caption to Fig. 2. 

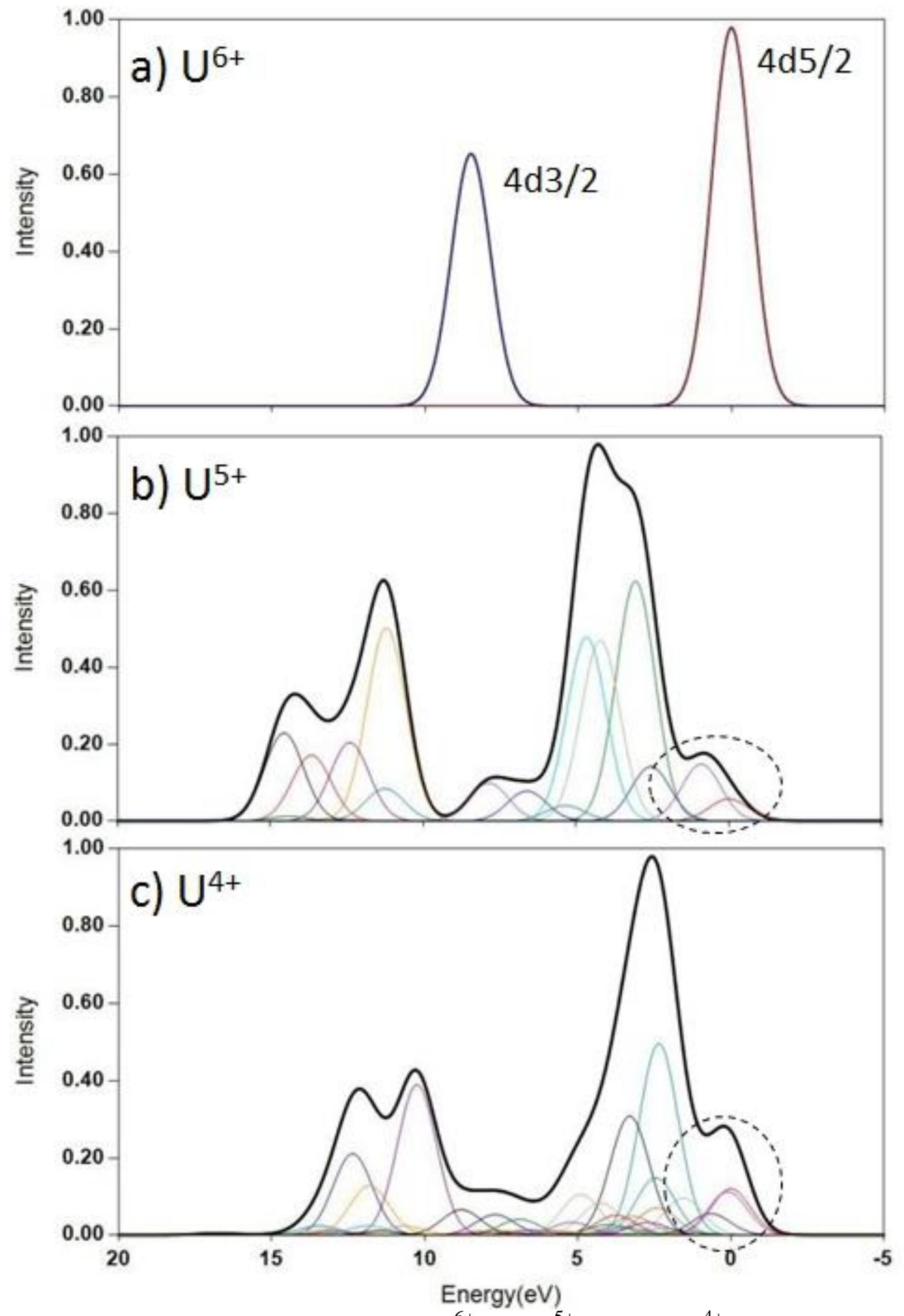

Figure 4. Plot of the 5d XPS for: (a) $\mathrm{U}^{6+}$, (b) $\mathrm{U}^{5+}$, and (c) $\mathrm{U}^{4+}$. See caption to Fig. 2. 

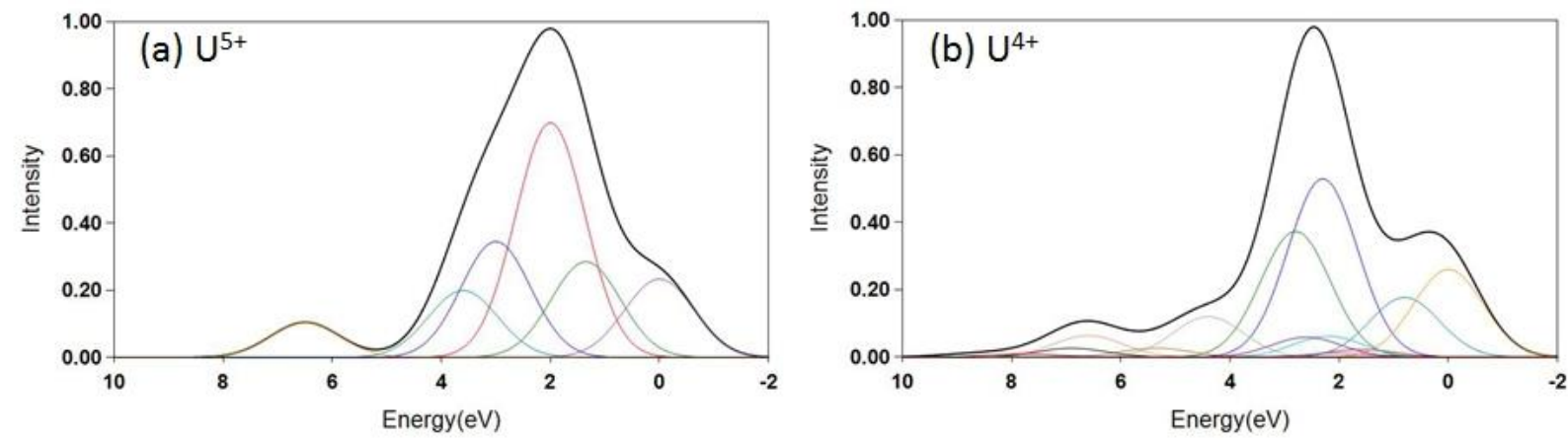

Figure 5. Plots of the 5 $\mathrm{p}_{3 / 2}$ XPS for: (a) $\mathrm{U}^{5+}$ and (b) $\mathrm{U}^{4+}$. See caption to Fig. 2. 

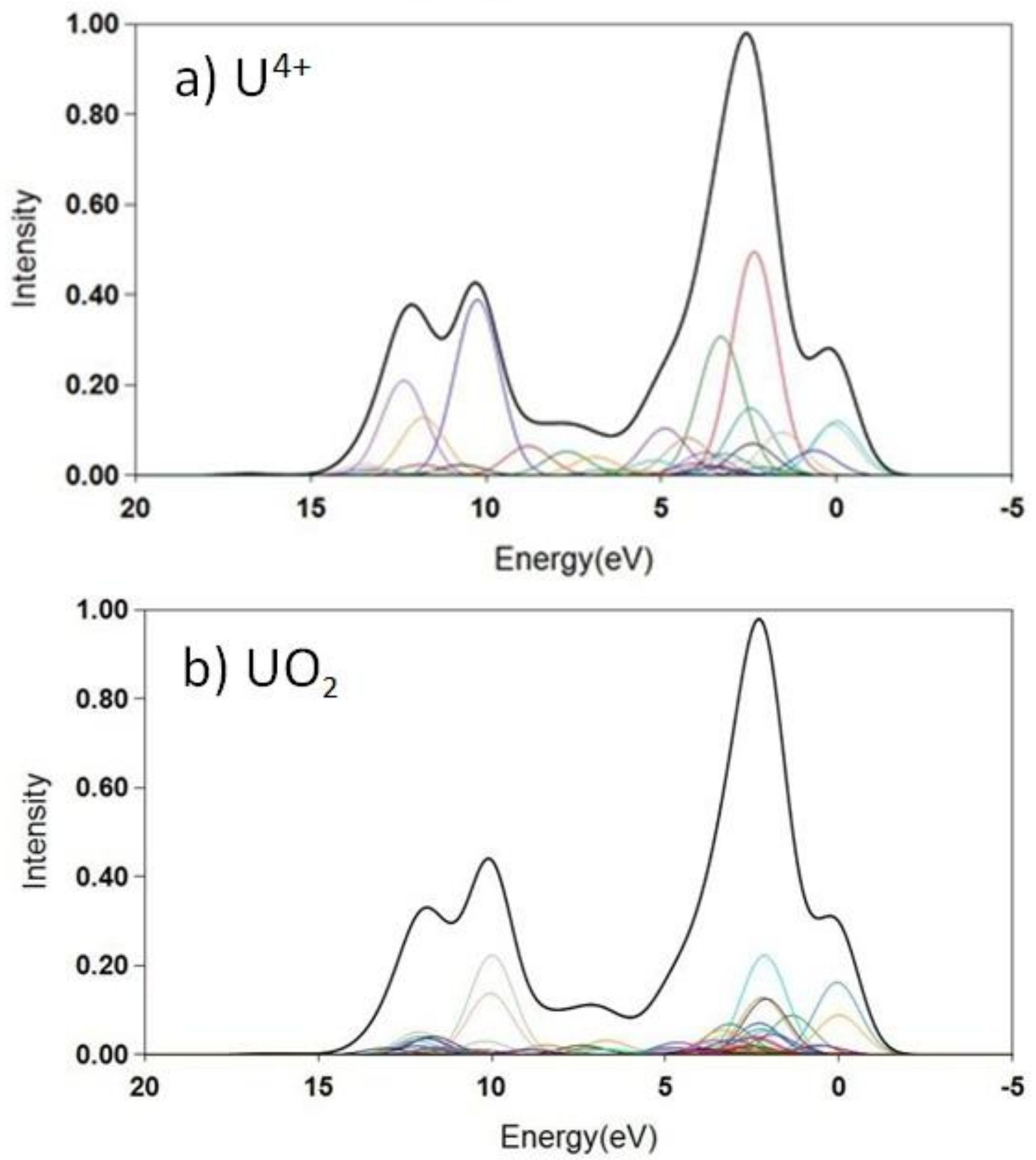

Figure 6. Comparison of the 5d XPS for: (a) $\mathrm{U}^{4+}$ and (b) $\mathrm{UO}_{2}$; see caption to Fig. 2. 
$\mathrm{Ce}^{3+}$ Atom - 4d XPS

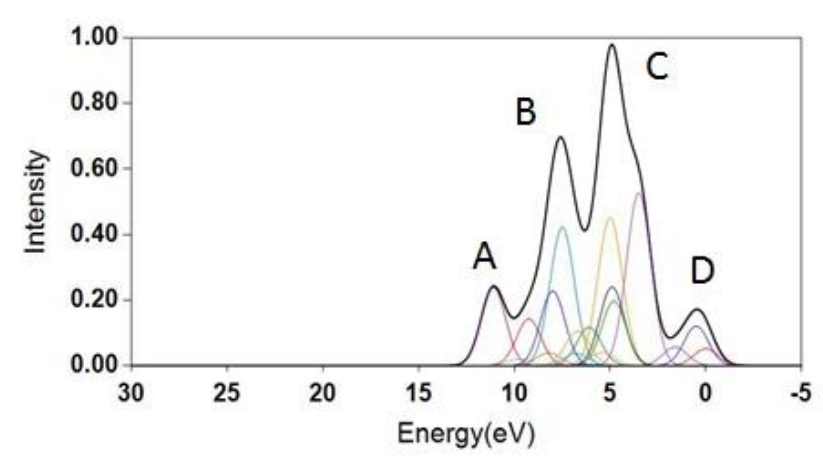

Mullins et al, Surface Science 429 (1999)

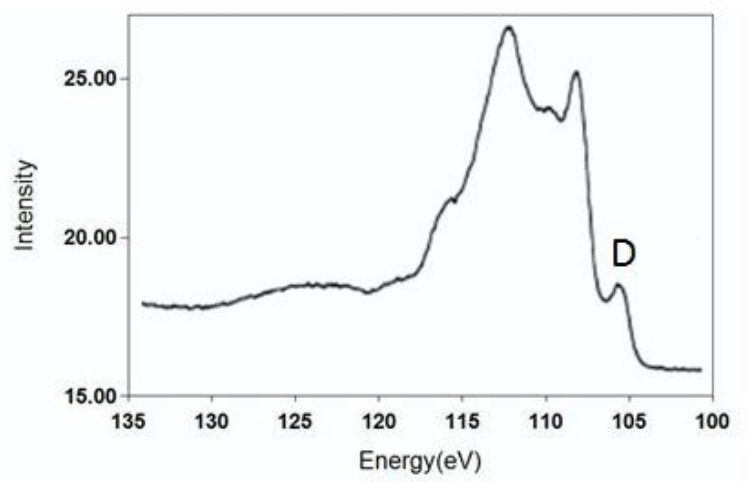

Figure 7. Comparison of the calculated $4 \mathrm{~d}$ XPS of $\mathrm{Ce}^{3+}$ with data for the measured $4 \mathrm{~d}$ XPS for $\mathrm{Ce}_{2} \mathrm{O}_{3}$ taken from Ref. [45]. The features of the theoretical spectra are labelled A-D and the label $\mathrm{D}$ is given to the low, $105 \mathrm{eV}, \mathrm{BE}$ feature of the experiment. See caption to Fig. 2.

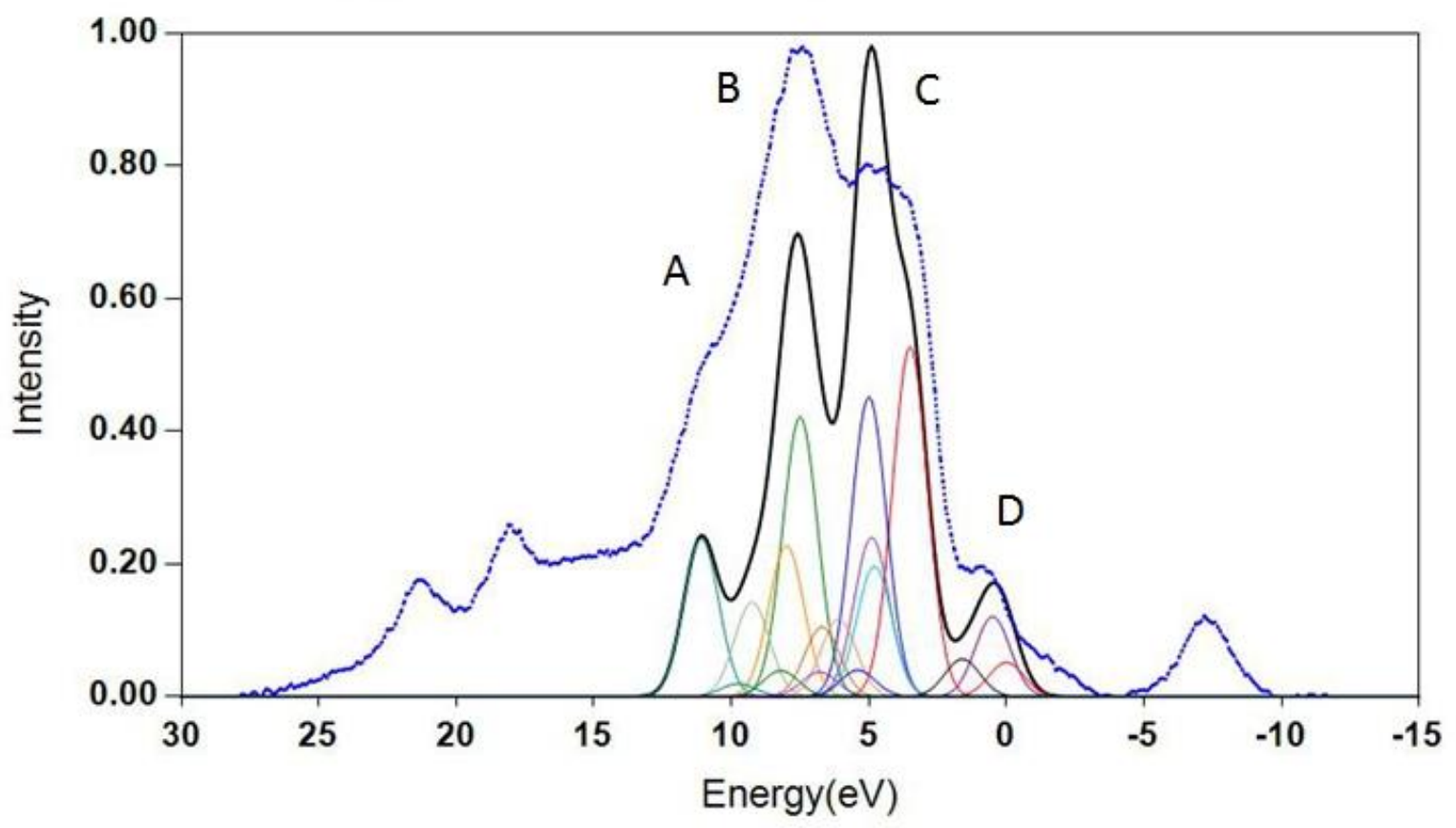

Figure 8. Comparison of the calculated $4 \mathrm{~d}$ XPS of $\mathrm{Ce}^{3+}$ with the measured $4 \mathrm{~d}$ XPS for $\mathrm{U}$ doped and sputtered $\mathrm{CeO}_{2}$; see text. The peaks are labelled as in Fig. 7. The theoretical peak B has been aligned with the maxima of the XPS for doped $\mathrm{CeO}_{2}$. See caption to Fig. 2 . 


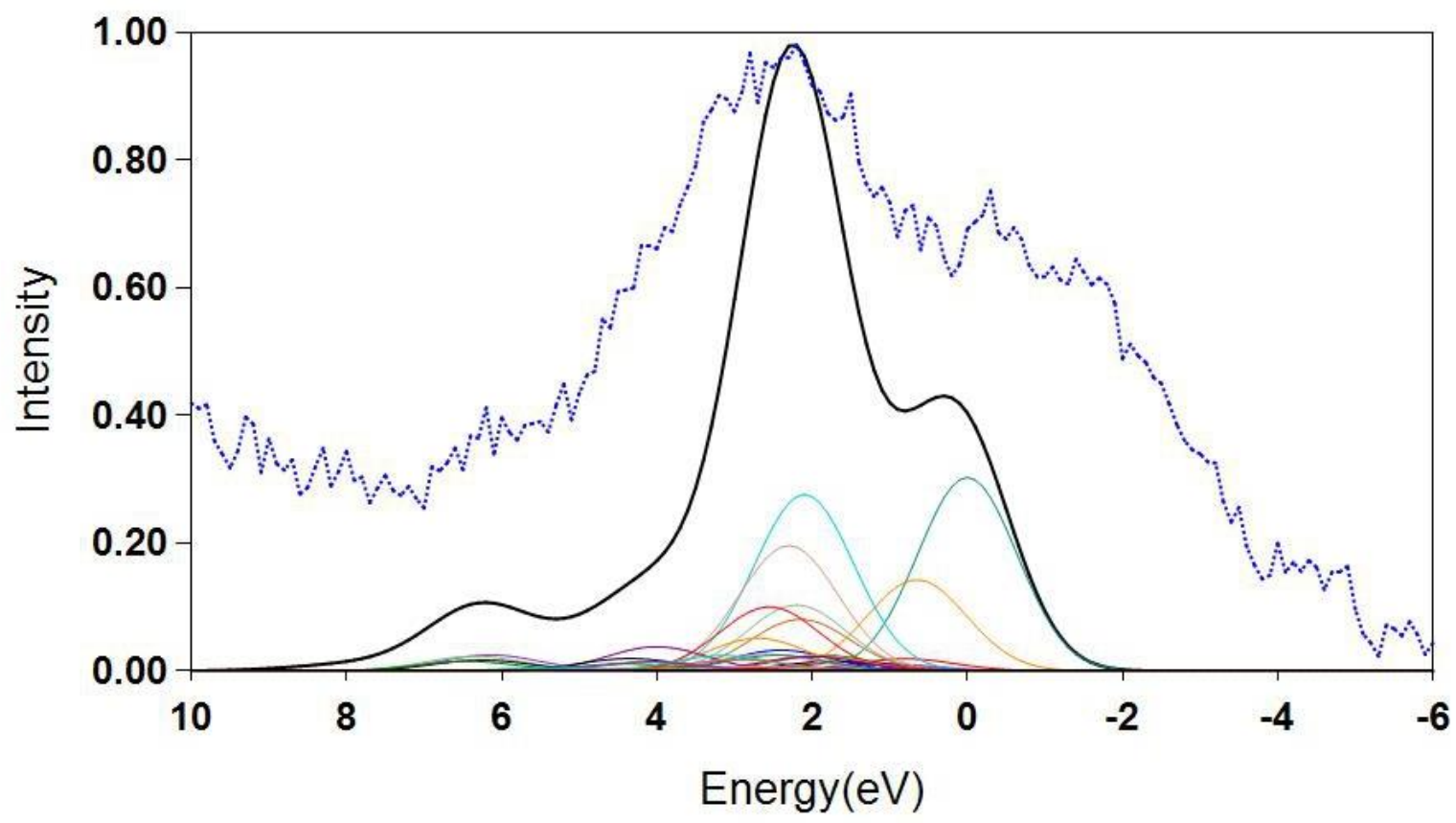

Figure 9. Comparison of the theoretical U 5 $\mathrm{p}_{3 / 2}$ XPS for the $\mathrm{UO}_{8}$ cluster model of $\mathrm{UO}_{2}$ with the measured $5 \mathrm{p}_{3 / 2}$ XPS for $\mathrm{UO}_{2}$; see caption to Fig. 2. The maxima of the main experimental and theoretical peaks are aligned. 


\section{Graphical Abstract}

The plots of the $3 \mathrm{~d}$ and $4 \mathrm{~d}$ XPS of closed shell $\mathrm{Ce}^{4+}$ and open shell $\mathrm{Ce}^{3+}$ show the strong dependence of the multiplet structure on the charge state and on the core shell ionized.
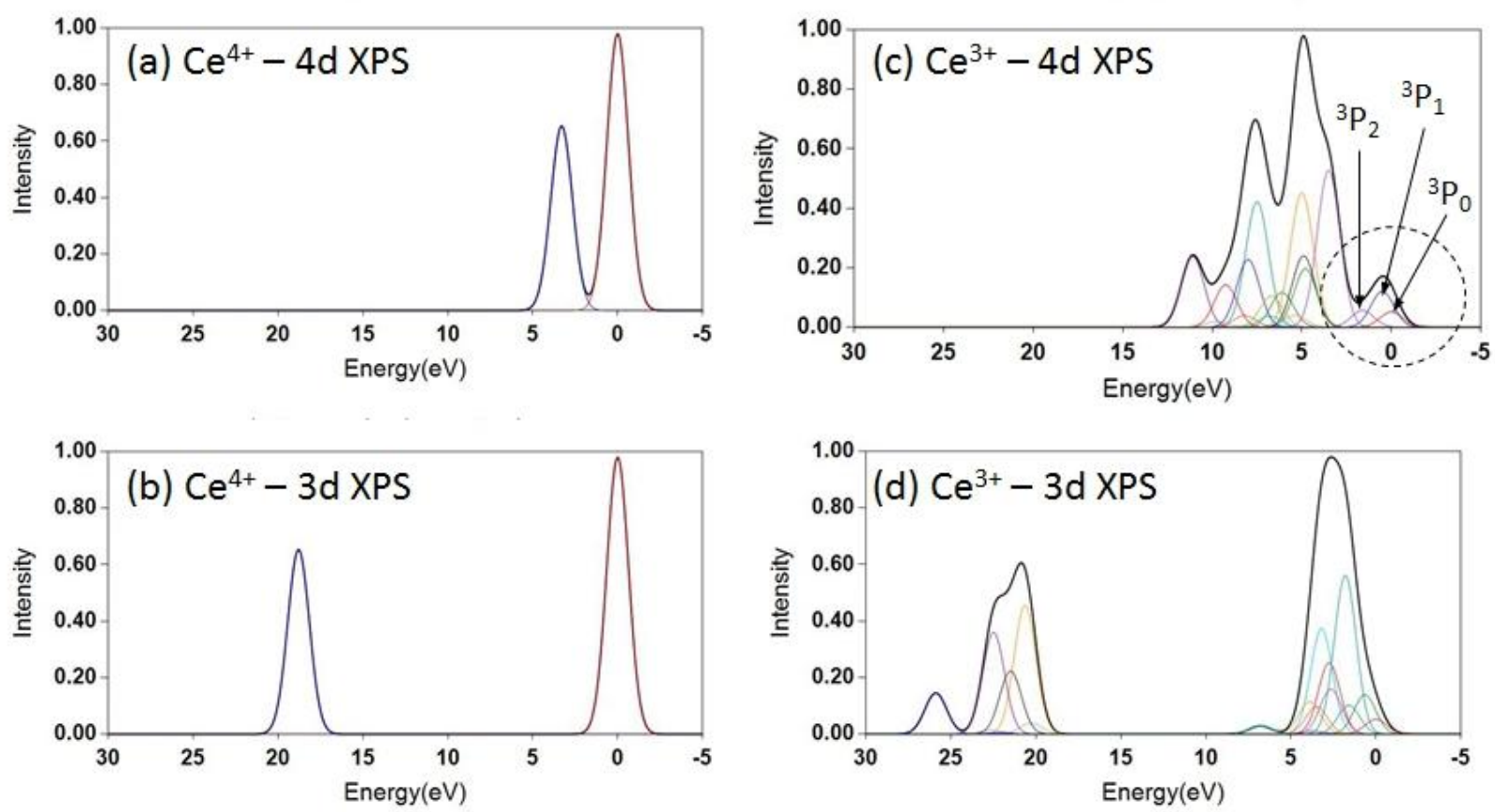